\title{
Transcriptional analysis of atrial and ventricular muscles from rats
}

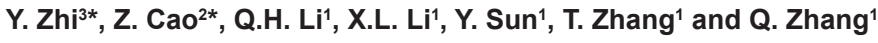 \\ 1Department of Cardiovascular Medicine, Changzhou TCM Hospital, \\ Changzhou, Jiangsu Province, China \\ ${ }^{2}$ Meng He Institute, Changzhou TCM Hospital, Changzhou, \\ Jiangsu Province, China \\ ${ }^{3}$ Nanjing University of Chinese Medicine, Xianlin University, \\ Nanjing, Jiangsu Province, China \\ *These authors contributed equally to this study. \\ Corresponding author: Q. Zhang \\ E-mail: zhangqidsds123@163.com \\ Genet. Mol. Res. 15 (1): gmr.15017330 \\ Received September 14, 2015 \\ Accepted November 9, 2015 \\ Published January 29, 2016 \\ DOI http://dx.doi.org/10.4238/gmr.15017330
}

ABSTRACT. Previous studies have used microarray technology to explore
gene expression differences between the atrium and the ventricle. However,
selection criteria for the differentially expressed genes (DEGs) based only
on either the fold change or the P value in these studies. Here, we aim to
further identify the DEGs by setting a P value threshold of $<0.05$ and a fold
change of $>2$, which may yield more specific gene expression differences
between the atrium and the ventricle. Gene expression profiling of the atrial
appendages and the ventricular free walls in 13 normal male Sprague
Dawley rats were obtained from the Gene Expression Omnibus data base
(accession No.: GSE5266). DEGs between the atrial and the ventricular
samples were screened using the microarray significance analysis. The
underlying functions of DEGs were predicted by gene ontology and pathway
enrichment analyses. In addition, we also constructed protein interactions
networks, and analyzed the function modules of the interacting proteins
by MCODE. A total of 757DEGs between the atria and the ventricles 
were found. The genes highly expressed in the ventricular myocytes were associated with muscle contraction (e.g., Myl1, Myl2, Myl3, and Myh7) and energy production (e.g., Acadm and Acs/6), while the genes preferentially expressed in the atrial myocytes were involved in the integration of neurohumoral signals (e.g., Cldn1). These conclusions were confirmed by pathway enrichment and function module analyses. Our present study provides an overview of the transcript level differences between the atrium and the ventricle, which may be useful for determination of potential biomarkers.

Key words: Brain natriuretic peptide; Cardiovascular disease; Gene expression profile; Functional association analysis; Diagnosis

\section{INTRODUCTION}

In mammals, the ventricles primarily generate contractile forces to pump the blood, while the atria, other than its contractile function, serve as a source and target for neurohumoral signals. In order to carry out these functions, the atrial and ventricular myocytes differ in morphology, ultrastructure, and gene expression (Legato, 1973; Forbes et al., 1990). For example, atrial cardiocytes synthesize and secrete more atrial natriuretic factor (Cantin et al., 1987) and phenylethanolamine- $N$-methyltransferase (Tillinger et al., 2006) as compared with ventricular cardiocytes. However, the calcium channel CaV1.2 (Larsen et al., 2002), the inwardly rectifying $\mathrm{K}^{+}$channel subunit Kir2.1 (Dhamoon et al., 2004), as well as the KCNE2 protein (Zhang et al., 2012) are more abundantly expressed in the ventricles than in the atria.

In addition, recent studies have also examined differences in gene expression between the atrium and the ventricle via microarray technology, which allows simultaneous determination of the expression levels of thousands of genes (Zhao et al., 2002; Tsubakihara et al., 2004; Barth et al., 2005; McGrath and de Bold., 2009). However, selection criteria for the differentially expressed genes (DEGs) are traditionally only based on either the fold change (FC) alone [>1.8 (McGrath and de Bold, 2009) or 2 (Tsubakihara et al., 2004)], or the adjusted $P$ value alone [false discovery rate (FDR) <0.03] (Barth et al., 2005). In this study, we aimed to further identify the DEGs between the atrium and the ventricle based on an FDR cut-off point of $<0.05$ and a FC of $>2\left(\left|\log _{2} F C\right|>1\right)$. This method may yield more genes that are specific for the atrium and the ventricle. In addition, we also performed functional analysis using various bioinformatic methods.

\section{MATERIAL AND METHODS}

\section{Data sets}

Gene expression profiling of the atrial appendages and the ventricular free walls in 13 male Sprague Dawley rats were downloaded from the Gene Expression Omnibus database (http:// www.ncbi.nlm.nih.gov/geo/) under the accession No. GSE5266 (McGrath and de Bold, 2009). The GeneChip platform used was the Rat Genome 230 2.0 Array from Affymetrix (Santa Clara, CA, USA). Tissues were grouped and divided into 4 pools to generate 4 biological replicates for each muscle type. All studies were approved by the University of Ottawa Animal Care Committee. 


\section{Data processing and statistical analysis}

The raw microarray data were pre-processed using the robust multiarray average analysis function included in the R/Bioconductor software, which performs normalization, background correction, and data summarization. DEGs between the atrial and the ventricular muscle samples were screened using the significance analysis of microarray method (Zhang, 2007), and the raw $\mathrm{P}$ values were adjusted according to FDR via the Benjamini \& Hochberg procedure (Shaffer, 1995). Genes were considered to be differentially expressed when FDR $<0.05$ and expression FC $>2$ $\left(\left|\log _{2} \mathrm{FC}\right|>1\right)$.

\section{Functional classification and gene annotation}

Screened genes from each group were classified into different functional categories through clusters of orthologous groups (COGs) (Tatusov et al., 2000; Tatusov et al., 2001; Tatusov et al., 2003). The COGs of proteins were generated by comparing the protein sequences of complete genomes. Each cluster contains proteins or groups of paralogs from at least three lineages. To obtain a better understanding of the biological functions of selected genes, gene ontology (GO) enrichment analyses were conducted using the Inter Pro Scan program. The selected genes were also mapped onto various pathways by the Gene Map Annotator and Pathway Profiler (GenMapp) software (Kanehisa et al., 2010).

\section{Protein-protein interactions and function module analysis}

Protein interactions between DEGs were predicted by STRING (http://string-db.org/) (Szklarczyk et al., 2011) with medium confidence, and the interaction network was analyzed by the Cytoscape 2.8.3 software (Shannon et al., 2003). The function modules of the interacting proteins were analyzed by MCODE (Bader and Hogue, 2003), and representation of these functional themes were mapped out by BiNGO (Maere et al., 2005), a plug-in of Cytoscape.

\section{RESULTS}

\section{DEGs between atrial and ventricular tissues}

Based on the pre-set threshold described above (FDR $<0.05$ and $\left.\left|\log _{2} \mathrm{FC}\right|>1\right), 757$ DEGs were identified between the atrial and ventricular tissues. Among them, 475 genes showed higher expression in the atrial samples, and 282 genes showed higher expression levels in the ventricular tissues. The significantly up- and down-regulated genes in ventricular tissues are listed in Table S1.

\section{Gene functional enrichment}

As shown in Table 1, DEGs were classified into several different functional categories. It was found that genes involved in signal transductions accounted for the largest proportion of the group, and involved 116 genes (22.14\%). Further functional enrichment analysis 
showed that overall, DEGs between atrial and ventricular muscles were mainly involved in the regulation of cell proliferation, vasculature development, and identical protein binding (Figure 1 and Table 1). In addition, we also performed pathway analysis for DEGs by using the Gene Map Annotator and Pathway Profiler (GenMapp) software. As a result, we identified 11 significant pathways (Table 2). Myl2 (myosin, light polypeptide 2, regulatory, cardiac, slow), Myl3 (myosin, light chain 3, alkali; ventricular, skeletal, slow) and Myh7 (myosin, heavy polypeptide 7, cardiac muscle, beta) genes were enriched in pathways involving cardiac muscle contraction (rno04260), hypertrophic cardiomyopathy (rno05410), leukocyte transendothelial migration (rno04670), and dilated cardiomyopathy (rno05414). In addition, genes regulating fatty acid metabolism (Acadm, acyl-Coenzyme A dehydrogenase, medium chain; Acs/6, acylCoA synthetase long-chain family member 6 ) and cell adhesion (Cldn1, claudin 1) were also enriched in the ventricular myocytes.

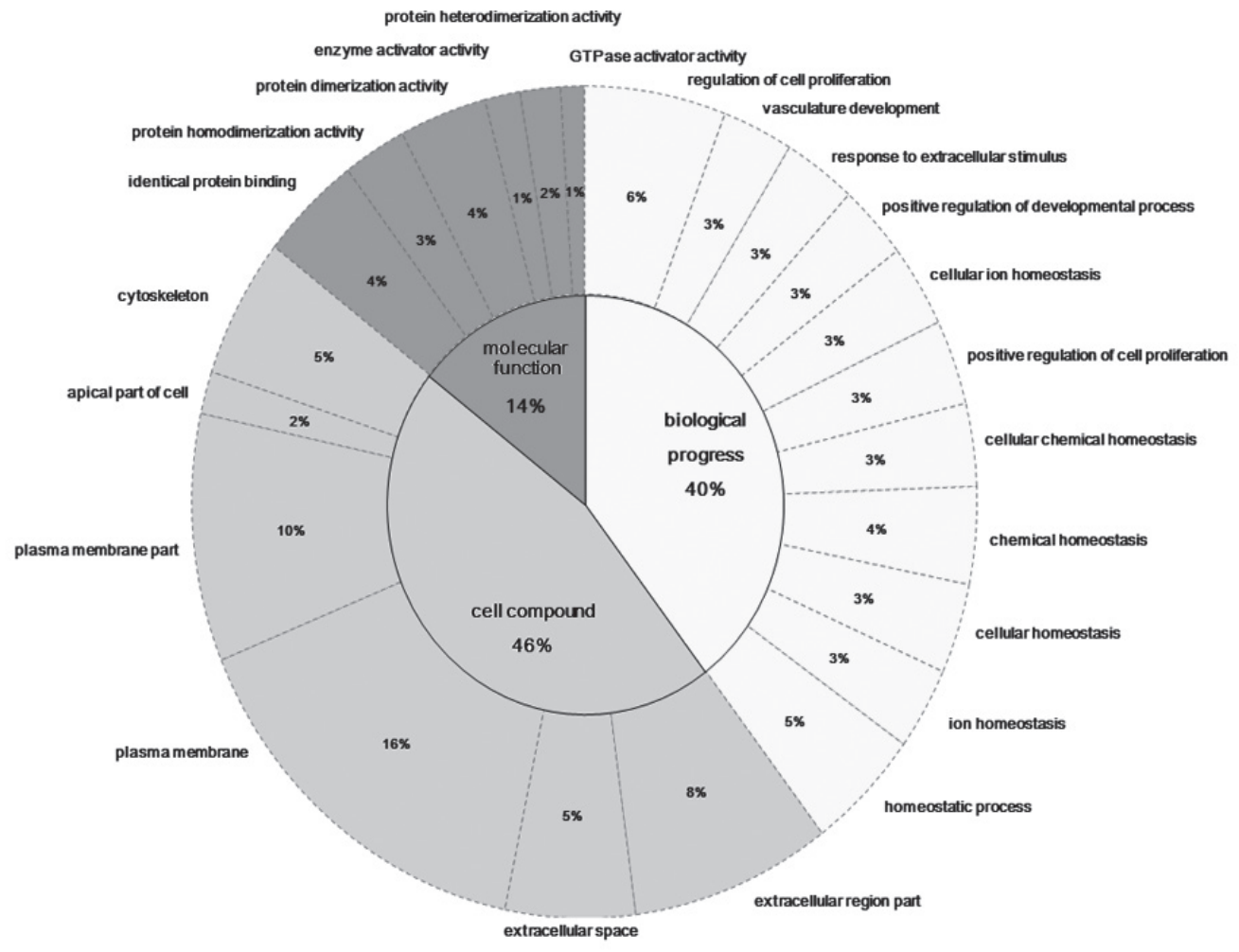

Figure 1. Functional classification of differentially expressed genes between the atria and the ventricles with known gene ontology annotations. White, gray, and black refer to biological progress, cell compound, and molecular function, respectively. Percentages in the figure denote differentially expressed genes plotted according to muscle type, i.e., atrium or ventricle. 
Table 1. COG categories of differentially expressed genes between the atria and the ventricles.

\begin{tabular}{|c|c|c|}
\hline Class definition & Number of class & Percent of class $(\%)$ \\
\hline Signal transduction mechanisms & 116 & 22.14 \\
\hline General function prediction only & 83 & 15.84 \\
\hline Transcription & 43 & 8.21 \\
\hline Cytoskeleton & 21 & 4.01 \\
\hline Function unknown & 40 & 7.63 \\
\hline Lipid transport and metabolism & 25 & 4.77 \\
\hline Extracellular structures & 16 & 3.05 \\
\hline Posttranslational modification, protein turnover, chaperones & 33 & 6.30 \\
\hline Carbohydrate transport and metabolism & 18 & 3.44 \\
\hline Energy production and conversion & 16 & 3.05 \\
\hline Inorganic ion transport and metabolism & 24 & 4.58 \\
\hline Amino acid transport and metabolism & 17 & 3.24 \\
\hline Secondary metabolites biosynthesis, transport and catabolism & 13 & 2.48 \\
\hline Cell cycle control, cell division, chromosome partitioning & 7 & 1.34 \\
\hline Nucleotide transport and metabolism & 5 & 0.95 \\
\hline Translation, ribosomal structure and biogenesis & 5 & 0.95 \\
\hline RNA processing and modification & 8 & 1.53 \\
\hline Chromatin structure and dynamics & 5 & 0.95 \\
\hline Intracellular trafficking, secretion, and vesicular transport & 16 & 3.053 \\
\hline Defense mechanisms & 5 & 0.95 \\
\hline Cell motility & 2 & 0.38 \\
\hline Coenzyme transport and metabolism & 2 & 0.38 \\
\hline Cell wall/membrane/envelope biogenesis & 2 & 0.38 \\
\hline Unclassified_Unclassified & 1 & 0.19 \\
\hline Replication, recombination and repair & 1 & 0.19 \\
\hline
\end{tabular}

Table 2. Enriched KEGG pathways for differentially expressed genes between the atria and ventricles.

\begin{tabular}{|c|c|c|}
\hline Term & $P$ value & Genes \\
\hline mo04260:Cardiac muscle contraction & 0.0019 & MYL2, MYL3, ATP1B4, ATP1A1, MYH7, COX6B2, CACNA2D3, CACNA1C, TPM2, L \\
\hline mo00071:Fatty acid metabolism & 0.0020 & ACAA2, ACADSB, ACADM, CPT2, ALDH1B1, ACADS, ALDH2, ACSL6 \\
\hline mo05410:Hypertrophic cardiomyopathy & 0.0032 & PRKAG3, ITGA9, MYL2, MYL3, PRKAG2, MYH7, CACNA2D3, CACNA1C, TPM2, CACNA1D, TGFB2 \\
\hline mo04512:ECM-receptor interaction & 0.0081 & ITGA9, LAMB3, COL4A2, COL4A1, CD44, LAMA5, VTN, SDC2, FN1, LOC681309 \\
\hline rno04670:Leukocyte transendothelial migration & 0.0109 & VCAM1, ITGAL, MYL7, EZR, MYL2, NCF1, CXCR4, CLDN1, CLDN11, VAV2, CXCL12, MYL9 \\
\hline mo04672:Intestinal immune network for lgA production & 0.0125 & CXCR4, RT1-DB1, RT1-DMB, RT1-BA, CXCL12, CCL27, TGFB2 \\
\hline mo00280:Valine, leucine and isoleucine degradation & 0.0138 & ACAA2, ACADSB, ACADM, ALDH1B1, ACADS, AOX1, ALDH2 \\
\hline mo00340:Histidine metabolism & 0.0186 & ASPA, ALDH1B1, ALDH1A3, ALDH2, ALDH3A1 \\
\hline 4:Cell adhesion molecules & 0.0268 & ITGAL, PTPRF, RT1-L \\
\hline cardiomyopathy & 0.04010 & ITGA9, MYL2, MYL3, MYH7, CACNA2 \\
\hline Ino04360:Axor & 0.0488 & SLIM1, EPHA4, PAK2, EFNB3, CXCR4, SEMA3G, ABLIM3, RHOD, FES, CXCL \\
\hline
\end{tabular}

\section{Protein-protein interactions and function module analysis}

Protein interactions between DEGs were predicted by STRING with medium confidence. An interaction network that included 644 interacting gene pairs was obtained (Figure 2). With the Cytoscape application and its plug-ins, we obtained functional modules of the interaction network, which is presented in Figure 3. In line with the pathway analysis, we also identified modules that were associated with cardiac muscle contraction, fatty acid metabolism, and cell adhesion.

\section{DISCUSSION}

According to our microarray data, 757 genes were identified to be differentially expressed in specific regions of the heart; 475 genes were highly expressed in the atrial samples, and 282 genes were more abundantly expressed in the ventricular tissues. Specifically, Myl1 was highly expressed in the atrial samples, while $M y l 2, M y l 3$, and Myh7 were highly expressed in the ventricular tissues. These results were in agreement with previous studies. Further functional and PPI network analyses suggested that Myl1, Myl2, Myl3, and Myh7 were important genes involved in muscle contraction. 


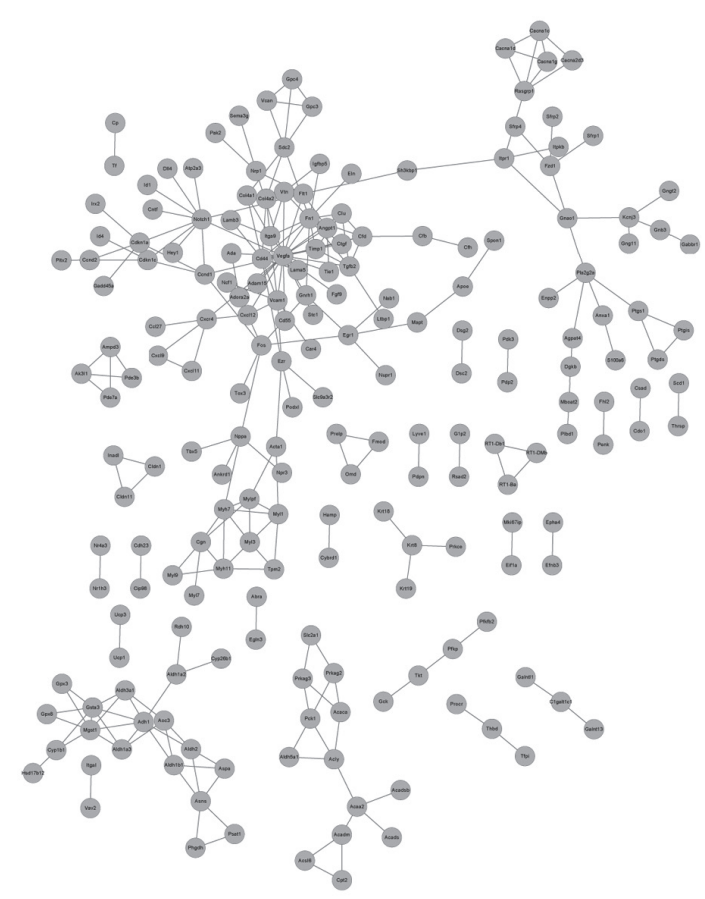

Figure 2. Interaction network of selected gene products. The circular nodes represent gene products, lines refer to interacting relationships between gene products.

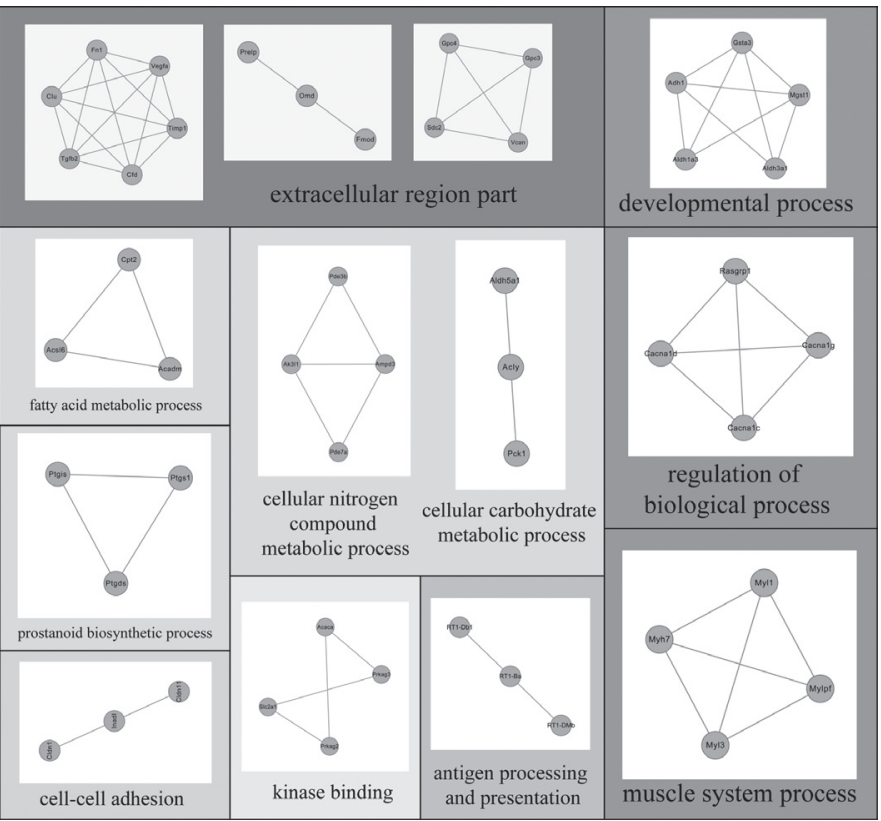

Figure 3. Functional modules of interaction network. 
Myosin is a hexameric enzyme, which consists of two pairs of light chains and two heavy chains. Myl2 makes up the regulatory light chain of myosin, while Myl3 makes up the essential light chain of myosin. The regulatory light chain binds $\mathrm{Ca}^{2+}$ at activating concentrations. In addition, phosphorylation of a serine residue in the $\mathrm{N}$-terminus of $M y / 2$ is also important for cardiac muscle contractions. In contrast to $M y / 2, M y / 3$ contains a unique $\mathrm{N}$-terminal domain that can bind actin, allowing Myl3 to contribute to force-generating myosin cross-bridges (Timson et al., 1998; Kazmierczak et al., 2009). Several studies have demonstrated that mutations of the Myl2 and Myl3 genes are associated with ventricular chamber-type cardiomyopathy (Irie et al., 2011). In addition, myosin light chain-2 has also been shown to be a specific marker for early ventricular hiPSC (human induced pluripotent stem cell)-derived cardiomyocytes, but not atrial hiPSC-derived cardiomyocytes (Bizy et al., 2013). Myh7gene encodes the cardiac $\beta$-myosin heavy chain, and is the predominant myosin isoform in the ventricular myocardium. Budde et al. (2007) have previously identified the missense mutationc. The $842 \mathrm{G}>\mathrm{Cin} M y h 7$, which affects a highly conserved amino acid in the myosin subfragment-1 (R281T). This mutation prevents salt bridge formation between residues R281 and D325, thereby destabilizing the myosin head (Budde et al., 2007). Richard et al. (2003) reported that changes to the Myh7 genes accounted for $82 \%$ of families with identified mutations, emphasizing its relevance for hypertrophic cardiomyopathy The Myl1 gene encodes the fast skeletal alkali myosin light polypeptide 1, and is a specific and early marker for development of fast skeletal muscle (Ling et al., 2010; Burguière et al., 2011). However, several studies also have indicated that Myl1 can be expressed in cardiac muscles including the atria and the left ventricle (Kelly et al., 1995; Franco et al., 1997). In this study, we found that Myl1 was more abundantly expressed in the atrial samples as compared with the ventricular tissues, which needs to be verified by future studies.

In addition, we also found that genes related to fatty acid metabolism (Acadm and Acs/6) and cell adhesion (Cldn1) were differentially expressed in the ventricles and the atria. Acyl-CoA dehydrogenase is an enzyme that catalyzes the first reactions of long-chain fatty acid $\beta$-oxidation in the mitochondria, and thus promotes ATP production in the heart (Le et al., 2000). Inherited deficiency of acyl-CoA dehydrogenase is associated with severe cardiac dysfunction including cardiac hypertrophy, polymorphic ventricular tachycardia, and cardiomyopathy, resulting in sudden death in neonates and children (Exil et al., 2003, 2006; Cox et al., 2009; Marcì and Ajovalasit, 2009). Acyl-CoA synthetase activates long chain fatty acids to form acyl-CoAs, and then directs fatty acids oxidation to generate ATP. Activation of fatty acids is catalyzed by a family of five long-chain acylCoA synthetases, namely, ACSL1, ACSL3, ACSL4, ACSL5, and ACSL6 (Ellis et al., 2010). In this study, we found that ACADM and ACSL6 were highly expressed in ventricular tissues, suggesting that energy metabolism occurs more prominently in ventricular cardiomyocytes. CLDN1 is an integral component of epithelial tight junctions, and overexpression of CLDN1 leads to cardiac looping (Simard et al., 2006). Previous studies have demonstrated that another tight junction protein, connexin 43 , is critical for migration of cardiac neural crest cells and the formation of peripheral ganglia (Huang et al., 1998). It was found that synaptic proteins were down-regulated in Cx43 null mouse hearts (lacobas et al., 2005). This finding suggests that tight junction proteins are associated with neuro humoral signals. Based on the proposed functions of the ventricles and the atria, CLDN1 was suggested to be highly expressed in the atria, which was confirmed by our study.

In conclusion, the present study provides insight into the differences in transcript levels between the atrium and the ventricle. We speculate that genes predominately expressed in the ventricular myocytes are associated with muscle contraction and energy production, while genes 
preferentially expressed in the atrial myocytes provide integration of neuro humoral signals. However, further studies are still required to confirm the function of these genes.

\section{Conflicts of interest}

The authors declare no conflicts of interest.

\section{REFERENCES}

Bader GD and Hogue CW (2003). An automated method for finding molecular complexes in large protein interaction networks. BMC Bioinformatics 4: 2.http://dx.doi.org/10.1186/1471-2105-4-2

Barth AS, Merk S, Arnoldi E, Zwermann L, et al. (2005). Functional profiling of human atrial and ventricular gene expression. Pflugers Arch. 450: 201-208.http://dx.doi.org/10.1007/s00424-005-1404-8

Bizy A, Guerrero-Serna G, Hu B, Ponce-Balbuena D, et al. (2013). Myosin light chain 2-based selection of human iPSC-derived early ventricular cardiac myocytes. Stem Cell Res. (Amst.) 11: 1335-1347.http://dx.doi.org/10.1016/j.scr.2013.09.003

Budde BS, Binner P, Waldmüller S, Höhne W, et al. (2007). Noncompaction of the ventricular myocardium is associated with a de novo mutation in the $\beta$-myosin heavy chain gene. PLoS One 2: e1362.http://dx.doi.org/10.1371/journal.pone.0001362

Burguière AC, Nord $\mathrm{H}$ and von Hofsten J (2011). Alkali-like myosin light chain-1 (myl1) is an early marker for differentiating fast muscle cells in zebrafish. Dev. Dyn. 240: 1856-1863.http://dx.doi.org/10.1002/dvdy.22677

Cantin M, Ding J, Thibault G, Gutkowska J, et al. (1987). Immunoreactive atrial natriuretic factor is present in both atria and ventricles. Mol. Cell. Endocrinol. 52: 105-113.http://dx.doi.org/10.1016/0303-7207(87)90103-1

Cox KB, Liu J, Tian L, Barnes S, et al. (2009). Cardiac hypertrophy in mice with long-chain acyl-CoA dehydrogenase or very long-chain acyl-CoA dehydrogenase deficiency. Lab. Invest. 89: 1348-1354.http://dx.doi.org/10.1038/labinvest.2009.86

Dhamoon AS, Pandit SV, Sarmast F, Parisian KR, et al. (2004). Unique Kir2.x properties determine regional and species differences in the cardiac inward rectifier K+ current. Circ. Res. 94: 1332-1339.http://dx.doi.org/10.1161/01. RES.0000128408.66946.67

Ellis JM, Li LO, Wu PC, Koves TR, et al. (2010). Adipose acyl-CoA synthetase-1 directs fatty acids toward b-oxidation and is required for cold thermogenesis. Cell Metab. 12: 53-64.http://dx.doi.org/10.1016/j.cmet.2010.05.012

Exil VJ, Roberts RL, Sims H, McLaughlin JE, et al. (2003). Very-long-chain acyl-coenzyme a dehydrogenase deficiency in mice. Circ. Res. 93: 448-455.http://dx.doi.org/10.1161/01.RES.0000088786.19197.E4

Exil VJ, Gardner CD, Rottman JN, Sims H, et al. (2006). Abnormal mitochondrial bioenergetics and heart rate dysfunction in mice lacking very-long-chain acyl-CoA dehydrogenase. Am. J. Physiol. Heart Circ. Physiol. 290: H1289-H1297.

Forbes MS, Van Niel EE and Purdy-Ramos SI (1990). The atrial myocardial cells of mouse heart: a structural and stereological study. J. Struct. Biol. 103: 266-279.http://dx.doi.org/10.1016/1047-8477(90)90045-E

Franco D, Kelly R, Lamers WH, Buckingham M, et al. (1997). Regionalized transcriptional domains of myosin light chain $3 f$ transgenes in the embryonic mouse heart: morphogenetic implications. Dev. Biol. 188: 17-33.http://dx.doi.org/10.1006/ $\underline{\text { dbio. } 1997.8622}$

Huang GY, Cooper ES, Waldo K, Kirby ML, et al. (1998). Gap junction-mediated cell-cell communication modulates mouse neural crest migration. J. Cell Biol. 143: 1725-1734.http://dx.doi.org/10.1083/jcb.143.6.1725

lacobas DA, lacobas S, LiWE, Zoidl G, et al. (2005). Genes controlling multiple functional pathways are transcriptionally regulated in connexin43 null mouse heart. Physiol. Genomics 20: 211-223.http://dx.doi.org/10.1152/physiolgenomics.00229.2003

Irie W, Murakami C, Nakamura S, Maeda K, et al. (2011). Analysis of ventricular myosin light chain genes in cardiomyopathy. Forensic Sci. Int.: Genet. Supplement Series 3: e143-e144.

Kanehisa M, Goto S, Furumichi M, Tanabe M, et al. (2010). KEGG for representation and analysis of molecular networks involving diseases and drugs. Nucleic Acids Res. 38: D355-D360.http://dx.doi.org/10.1093/nar/gkp896

Kazmierczak K, Xu Y, Jones M, Guzman G, et al. (2009). The role of the N-terminus of the myosin essential light chain in cardiac muscle contraction. J. Mol. Biol. 387: 706-725.http://dx.doi.org/10.1016/j.jmb.2009.02.006

Kelly R, Alonso S, Tajbakhsh S, Cossu G, et al. (1995). Myosin light chain 3F regulatory sequences confer regionalized cardiac and skeletal muscle expression in transgenic mice. J. Cell Biol. 129: 383-396.http://dx.doi.org/10.1083/jcb.129.2.383

Larsen JK, Mitchell JW and Best PM (2002). Quantitative analysis of the expression and distribution of calcium channel alpha 1 subunit mRNA in the atria and ventricles of the rat heart. J. Mol. Cell. Cardiol. 34: 519-532.http://dx.doi.org/10.1006/ imcc.2001.1534

Le W, Abbas AS, Sprecher H, Vockley J, et al. (2000). Long-chain acyl-CoA dehydrogenase is a key enzyme in the 
mitochondrial $\beta$-oxidation of unsaturated fatty acids. Biochim. Biophys. Acta, Mol. Cell Biol. Lipids 1485: 121-128. http:/l dx.doi.org/10.1016/S1388-1981(00)00034-2

Legato MJ (1973). Ultrastructure of the atrial, ventricular, and Purkinje cell, with special reference to the genesis of arrhythmias. Circulation 47: 178-189.http://dx.doi.org/10.1161/01.CIR.47.1.178

Ling F, Fang W, Chen Y, Li J, et al. (2010). Identification of novel transcripts from the porcine MYL1 gene and initial characterization of its promoters. Mol. Cell. Biochem. 343: 239-247.http://dx.doi.org/10.1007/s11010-010-0519-1

Maere S, Heymans K and Kuiper M (2005). BiNGO: a Cytoscape plugin to assess overrepresentation of gene ontology categories in biological networks. Bioinformatics 21: 3448-3449.http://dx.doi.org/10.1093/bioinformatics/bti551

Marcì M and Ajovalasit P (2009). Medium-chain acyl-CoA dehydrogenase deficiency in an infant with dilated cardiomyopathy. Cardiol. Res. Pract. 2009: 281389.http://dx.doi.org/10.4061/2009/281389

McGrath MF and de Bold AJ (2009). Transcriptional analysis of the mammalian heart with special reference to its endocrine function. BMC Genomics 10: 254.http://dx.doi.org/10.1186/1471-2164-10-254

Richard P, Charron P, Carrier L, Ledeuil C, et al.; EUROGENE Heart Failure Project (2003). Hypertrophic cardiomyopathy: distribution of disease genes, spectrum of mutations, and implications for a molecular diagnosis strategy. Circulation 107: 2227-2232.http://dx.doi.org/10.1161/01.CIR.0000066323.15244.54

Shaffer JP (1995). Multiple hypothesis testing. Annu. Rev. Psychol. 46: 561-584. http://dx.doi.org/10.1146/annurev. ps.46.020195.003021

Shannon P, Markiel A, Ozier O, Baliga NS, et al. (2003). Cytoscape: a software environment for integrated models of biomolecular interaction networks. Genome Res. 13: 2498-2504.http://dx.doi.org/10.1101/gr.1239303

Simard A, Di Pietro E, Young CR, Plaza S, et al. (2006). Alterations in heart looping induced by overexpression of the tight junction protein Claudin-1 are dependent on its C-terminal cytoplasmic tail. Mech. Dev. 123: 210-227.http://dx.doi. org/10.1016/j.mod.2005.12.004

Szklarczyk D, Franceschini A, Kuhn M, Simonovic M, et al. (2011). The STRING database in 2011: functional interaction networks of proteins, globally integrated and scored. Nucleic Acids Res. 39: D561-D568.http://dx.doi.org/10.1093/nar/gkq973

Tatusov RL, Galperin MY, Natale DA and Koonin EV (2000). The COG database: a tool for genome-scale analysis of protein functions and evolution. Nucleic Acids Res. 28: 33-36.http://dx.doi.org/10.1093/nar/28.1.33

Tatusov RL, Natale DA, Garkavtsev IV, Tatusova TA, et al. (2001). The COG database: new developments in phylogenetic classification of proteins from complete genomes. Nucleic Acids Res. 29: 22-28.http://dx.doi.org/10.1093/nar/29.1.22

Tatusov RL, Fedorova ND, Jackson JD, Jacobs AR, et al. (2003). The COG database: an updated version includes eukaryotes. BMC Bioinformatics 4: 41.http://dx.doi.org/10.1186/1471-2105-4-41

Tillinger A, Bruderova V, Kubovcakova L, Zeman M, et al. (2006). Gene expression of the phenylethanolamine $\mathrm{N}$-methyltransferase is differently modulated in cardiac atria and ventricles. Gen. Physiol. Biophys. 25: 355-364.

Timson DJ, Trayer HR and Trayer IP (1998). The N-terminus of A1-type myosin essential light chains binds actin and modulates myosin motor function. Eur. J. Biochem. 255: 654-662.http://dx.doi.org/10.1046/j.1432-1327.1998.2550654.x

Tsubakihara M, Williams NK, Keogh A and dos Remedios CG (2004). Comparison of gene expression between left atria and left ventricles from non-diseased humans. Proteomics 4: 261-270.http://dx.doi.org/10.1002/pmic.200300539

Zhang M, Wang Y, Jiang M, Zankov DP, et al. (2012). KCNE2 protein is more abundant in ventricles than in atria and can accelerate hERG protein degradation in a phosphorylation-dependent manner. Am. J. Physiol. Heart Circ. Physiol. 302: H910-H922.http://dx.doi.org/10.1152/ajpheart.00691.2011

Zhang S (2007). A comprehensive evaluation of SAM, the SAM R-package and a simple modification to improve its performance. BMC Bioinformatics 8: 230.http://dx.doi.org/10.1186/1471-2105-8-230

Zhao XS, Gallardo TD, Lin L, Schageman JJ, et al. (2002). Transcriptional mapping and genomic analysis of the cardiac atria and ventricles. Physiol. Genomics 12: 53-60.http://dx.doi.org/10.1152/physiolgenomics.00086.2002

\section{Supplementary material}

Table S1. COG categories of differentially expressed genes between the atria and the ventricles.

http://www.geneticsmr.com/year2016/vol15-1/pdf/gmr7330_supplementary.pdf 